\title{
Seeing Is Believing: Effects of Visual Contextual Cues on Learning and Transfer of Locomotor Adaptation
}

\author{
Gelsy Torres-Oviedo and Amy J. Bastian \\ Motion Analysis Laboratory, Kennedy Krieger Institute, Baltimore, Maryland 21205, and Department of Neuroscience, The Johns Hopkins University \\ School of Medicine, Baltimore, Maryland 21205
}

\begin{abstract}
Devices such as robots or treadmills are often used to drive motor learning because they can create novel physical environments. However, the learning (i.e., adaptation) acquired on these devices only partially generalizes to natural movements. What determines the specificity of motor learning, and can this be reliably made more general? Here we investigated the effect of visual cues on the specificity of split-belt walking adaptation. We systematically removed vision to eliminate the visual-proprioceptive mismatch that is a salient cue specific to treadmills: vision indicates that we are not moving while leg proprioception indicates that we are. We evaluated the adaptation of temporal and spatial features of gait (i.e., timing and location of foot landing), their transfer to walking over ground, and washout of adaptation when subjects returned to the treadmill. Removing vision during both training (i.e., on the treadmill) and testing (i.e., over ground) strongly improved the transfer of treadmill adaptation to natural walking. Removing vision only during training increased transfer of temporal adaptation, whereas removing vision only during testing increased the transfer of spatial adaptation. This dissociation reveals differences in adaptive mechanisms for temporal and spatial features of walking. Finally training without vision increased the amount that was learned and was linked to the variability in the behavior during adaptation. In conclusion, contextual cues can be manipulated to modulate the magnitude, transfer, and washout of device-induced learning in humans. These results bring us closer to our ultimate goal of developing rehabilitation strategies that improve movements beyond the clinical setting.
\end{abstract}

\section{Introduction}

Movement naturally occurs in many different environments (e.g., water, snow), which create distinct challenges for the motor system. Through experience we acquire a repertoire of different sensorimotor calibrations, or internal models, that can be called on for different situations (for example, see Wolpert and Kawato, 1998; Haruno et al., 2001; Imamizu et al., 2003; Lee and Schweighofer, 2009). The brain must "choose" the best internal model for the situation at hand based on the available contextual information, prior experience, and the state of the body.

Upon experiencing changes in the environment or the body, the chosen internal model may have to be adapted to best perform within the new demands for movement. This adaptation process updates a neural representation of the body, the environment, or their interaction, used for a specific situation (Wolpert et al., 1995, 1998). The extent to which adaptation effects transfer to other situations reflects the overlap in the neural representations that are being used. Full transfer from one context to another would suggest that the same internal model is being used in both situations, whereas no transfer would suggest the use of separate internal models.

Recent work has shown that adaptation driven by devices (e.g., a treadmill or robot) is rather specific, showing limited

Received Aug. 11, 2010; revised Oct. 6, 2010; accepted 0ct. 18, 2010.

This work was supported by National Institutes of Health Grant R01 HD048741. We acknowledge N. H. Bhanpuri for insightful conversations during the preparation of this manuscript.

Correspondence should be addressed to Amy J. Bastian, Kennedy Krieger Institute, Motion Analysis Laboratory, Room G-04, 707 N. Broadway, Baltimore, MD 21205. E-mail: bastian@kennedykrieger.org.

DOI:10.1523/JNEUROSCI.4205-10.2010

Copyright $\odot 2010$ the authors $\quad 0270-6474 / 10 / 3017015-08 \$ 15.00 / 0$ transfer to natural movements without the device (Cothros et al., 2006; Kluzik et al., 2008; Reisman et al., 2009). Further, natural movements do not washout the stored calibration for the device: subjects show large adaptation effects that remain after making movements without the device (Reisman et al., 2009). Thus, the nervous system appears to be capable of forming a device-specific internal model and choosing a different internal model when performing the same movement off of the device.

How does the nervous system choose between different internal models for movement control? Contextual cues are useful for signaling a change in the choice of internal model-this has been shown for arm (Cothros et al., 2009), wrist (Osu et al., 2004), and eye movements (Shelhamer et al., 2005; Herman et al., 2009). These studies focused on how internal models could be kept separate; here we ask whether we can manipulate context cues to improve transfer of adaptation from one situation to another.

We tested whether removing visual cues improves transfer of the learned walking pattern from the split-belt treadmill to natural walking. We reasoned that visual cues are particularly salient during treadmill walking since they signal no motion in spacethis is at odds with proprioceptive signals from the legs and creates a mismatch in sensory information not experienced during natural walking. Eliminating this sensory mismatch may make the treadmill and natural walking contexts more similar and therefore may increase the transfer of learning between the two. We further asked whether visual cues have more of an effect on transfer of spatial (i.e., where to step) versus temporal (i.e., when to step) elements of the walking pattern. Since vision can be used to explicitly modify spatial adaptation (Malone and Bastian, 2010). 


\section{A Overall paradigm}

\begin{tabular}{|c|c|c|c|}
\hline $\mathrm{BAS}$ & EIINE & ADAPTATION & POST-ADAPTATION \\
\hline $\begin{array}{l}\text { Over } \\
\text { ground }\end{array}$ & Treadmill & Treadmill split-belts & $\begin{array}{c}\text { Over } \\
\text { ground }\end{array}$ \\
\hline
\end{tabular}

B Belt speeds for experiments 1 and 2

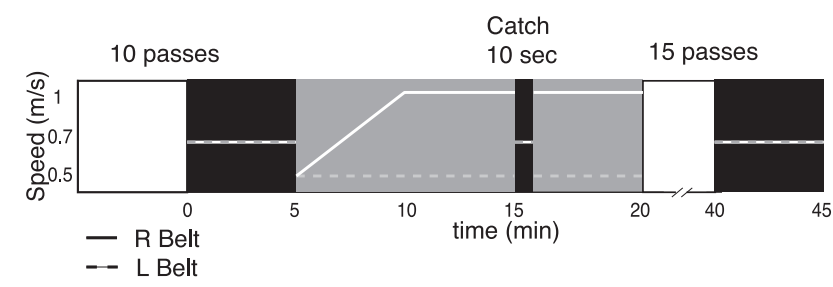

Figure 1. Overall paradigm and perturbation speeds. $A$, In all groups, baseline behavior was recorded over ground and subsequently on the treadmill. Then subjects were adapted for a total of $15 \mathrm{~min}$. A 10 s catch trial was introduced when subjects had been adapted for $10 \mathrm{~min}$. Subjects were readapted for 5 more minutes before they were asked to walk over ground, where we tested the transfer of treadmill adaptation to natural walking. Finally, subjects returned to the treadmill where they walked for 5-10 min to determine the washout of learning specific to the treadmill from the remaining aftereffects. $\boldsymbol{B}$, Belt speeds for all experiments. During baseline periods on the treadmill, both belts were moving at $0.7 \mathrm{~m} / \mathrm{s}$. To facilitate adaptation without vision, the belt speed ratio was gradually increased during the first $5 \mathrm{~min}$. Specifically, the speed of the right belt was gradually increased to $1 \mathrm{~m} / \mathrm{s}$ during the first $5 \mathrm{~min}$ and the speed of the other belt was maintained at $0.5 \mathrm{~m} / \mathrm{s}$. The two belts moved at the same speed as in baseline during the $10 \mathrm{~s}$ catch trial. Then subjects were readapted by maintaining the belts' ratio at 2:1. Finally, during the postadaptation on the treadmill belts were set back to the tied mode at 0.7 $\mathrm{m} / \mathrm{s}$. During baseline and postadaptation periods over ground, subjects walked multiple backand-forward passes on a $6 \mathrm{~m}$ walkway. All steps on the walkway were recorded except for those when subjects were turning to return to the initial position.

\section{Materials and Methods}

Thirty-nine healthy adults participated in this study. Twenty-three subjects ( 7 males and 16 females; mean age $25.2 \pm 4.9$ years) participated in experiment 1 and 16 subjects ( 5 males and 11 females; mean age $23 \pm 5.9$ years) participated in experiment 2 . The experimental protocol was approved by the Institutional Review Board at the Johns Hopkins University School of Medicine and all subjects gave informed consent before testing.

\section{Paradigm}

Subjects adapted their walking pattern on a split-belt treadmill, and we tested the transfer of this learning to overground walking (i.e., off of the treadmill). Locomotor adaptation was achieved using a split-belt treadmill (Woodway USA) that drove the speed of each leg independently. This paradigm has been demonstrated to induce the storage of a modified walking pattern that is expressed as an aftereffect in regular walking conditions and must be de-adapted to return to normal walking (Reisman et al., 2005). The overall paradigm for all experiments is illustrated in Figure $1 \mathrm{~A}$. In all experiments, subjects walked without holding on to a handrail while on the treadmill. We stretched bungee cords in front of, behind, and to the sides of the subject at midcalf height to keep subjects positioned on the treadmill (particularly subjects walking without vision). This gave subjects tactile cues for when they were moving too far in any given direction but was compliant so that it did not provide a solid "ground-referenced" cue. We collected a baseline period before adaptation in which subjects walked for $2-5 \mathrm{~min}$ on the treadmill with the belts moving together (i.e., "tied") at $0.7 \mathrm{~m} / \mathrm{s}$. Subjects then walked during an adaptation period for a total of $15 \mathrm{~min}$. During the first $5 \mathrm{~min}$ of adaptation the belt under the left leg moved at $0.5 \mathrm{~m} / \mathrm{s}$ while the belt under the right leg linearly increased its speed from $0.5 \mathrm{~m} / \mathrm{s}$ to $1.0 \mathrm{~m} / \mathrm{s}$ (Fig. $1 B$ ). Belts were then maintained at a 2:1 ratio for 5 more minutes before a $10 \mathrm{~s}$ "catch" period in which both belts were moving at the same speed $(0.7$ $\mathrm{m} / \mathrm{s}$; same speed as in the baseline period) (Fig. $1 B$ ). The recordings during this catch period allowed us to assess storage of the adaptation effects (i.e., aftereffects) on the treadmill. Subjects then walked in the 2:1 split-belt condition for an additional 5 min to readapt the walking pattern. After the entire adaptation period, subjects were transported on a wheelchair from the treadmill to a $6 \mathrm{~m}$ walkway were the overground transfer was tested. Subjects walked on the walkway for 15 back-andforward passes to test for transfer to overground walking of aftereffects due to the split-belt treadmill adaptation. Subjects were asked not to step when sitting on the wheelchair and when standing up to record as many of their initial steps after split-belt treadmill adaptation. Although the self-selected walking speeds in all subjects ranged between $0.6 \mathrm{~m} / \mathrm{s}$ and 1 $\mathrm{m} / \mathrm{s}$, the average walking speeds across experimental groups was not significantly different $\left(F_{(3,35)}=0.85, p=0.48\right)$. After assessing overground transfer, subjects returned to the treadmill and walked for 5-10 min in the tied-belts condition at $0.7 \mathrm{~m} / \mathrm{s}$. (Fig. $1 B$ ). This last period allowed us to test for washout of the treadmill aftereffects due to overground walking. The treadmill was stopped and restarted again at every speed transition.

We designed two experiments to test how visual context cues affect transfer of split-belt walking adaptation. In experiment 1 , visual feedback was removed in the training (i.e., treadmill) and testing (i.e., over ground) environments. This was done to assess whether visual context cues could modulate 1) the transfer of adaptation from one situation to another and 2) the washout of adaptation when returning to the training environment. In experiment 2 , visual feedback was removed in either the training or the testing environment to determine the degree to which the visual context cues during training or testing mediated the transfer and washout of adaptation. Spatial and temporal features of gait were analyzed in all experiments to identify possible differential effects of visual feedback on these two features of locomotion.

Experiment 1: transfer without vision. We tested how vision affects the magnitude of adaptation, transfer to overground walking, and subsequent washout of the adapted pattern on the treadmill. Two groups were compared: the vision $(n=8)$ and no-vision $(n=8)$ groups. The vision and no-vision group walked with or without vision, respectively, during all phases of the experiment. Subjects in the no-vision group only opened their eyes during the turns on the walkway, when they returned to the initial position. The no-vision group was given extra time at baseline ( 5 $\mathrm{min}$ ) so that they were comfortable walking on the treadmill with eyes closed. Also, as a control we added the no-vision catch group $(n=7)$, in which subjects walked with vision during adaptation and postadaptation but were tested with no vision during the catch trial. This group was used to determine whether any differences in aftereffects during the catch trial were due to sensory conditions during adaptation (training) and not simply the fact that the eyes were closed during the catch trial.

Experiment 2: transfer without vision in training or testing. Here we asked whether the effect of vision on transfer occurs due to its presence or absence during the adaptation (i.e., training) period, or during the over ground (i.e., testing) period. Two groups were compared: a no-vision training group $(n=8)$ and no-vision testing group $(n=8)$. The novision training group adapted on the split-belt treadmill with eyes closed, but was tested for overground generalization with eyes open. The novision testing group did the opposite- they trained with eyes open but were tested over ground with eyes closed. The transfer and washout of these two groups was compared with that of groups in experiment 1 . The no-vision catch group walked with eyes open on the treadmill (during adaptation and washout) but with the eyes closed over ground, as the no-vision testing group.

\section{Data collection}

Kinematic data were collected at $100 \mathrm{hz}$ using Optotrak (Northern Digital). Infrared-emitting markers were placed bilaterally over the following joints: foot (fifth metatarsal head), ankle (lateral malleolus), knee (lateral femoral epicondyle), hip (greater trochanter), pelvis (iliac crest) and shoulder (acromion process). The location of these makers is illustrated in Figure $2 \mathrm{~A}$. The times of heel strike and toe off (i.e., when the foot contacts and lifts off the ground) were recorded by foot-switches placed on the bottom of the shoes or were estimated from the ankle kinematic data. In all experiments subjects were instructed to walk with their arms 


\section{A Step symmetry (Spatial)}

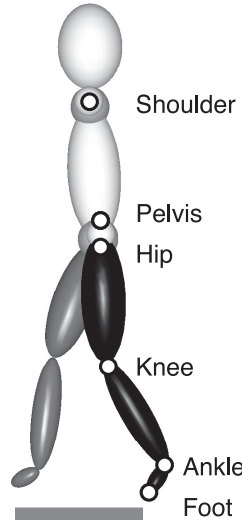

Step length 1
Step length 2

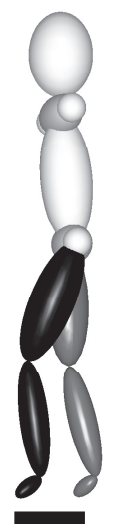

\section{B Phase shift symmetry (Temporal)}
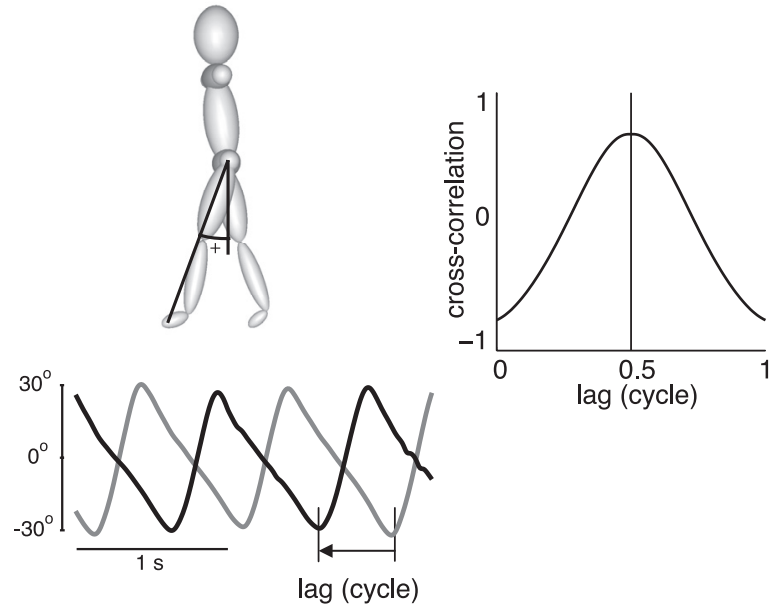

Figure 2. Spatial and temporal parameters to quantify gait symmetry. A, Spatial symmetry was quantified by the normalized difference in step lengths. Figurines were made from kinematic data of two consecutive steps during catch trial of a sample subject. Spatial aftereffects are illustrated by the difference in step lengths when the right leg (gray) is leading versus when the left leg is leading (black). $\boldsymbol{B}$, Temporal symmetry was quantified by the phase shift (lag) producing the largest cross-correlation between the right and left limb angles. Behavior during baseline is shown. Limb angle is defined as the angle between the limb axis (line from hip to ankle marker) and the vertical axis, as shown on the axis drawn on the top-left figurine. Example of limb angle trajectories as a function of time are shown at the bottom-left (gray and black oscillatory traces at the bottom). Top-right panel indicates the cross-correlation values as a function of lag for the cycles shown on the left. During baseline, legs move in anti-phase; consequently a lag of 0.5 leads to the maximal cross-correlation value.

crossed to allow for data collection without occlusion of hip and pelvis markers.

\section{Data analysis}

Spatial and temporal characteristics of gait that are known to adapt during split-belt treadmill walking were assessed (Choi and Bastian, 2007). The spatial parameter was step symmetry, which is defined as the difference between step lengths of the two legs (step length $=$ distance between two ankle markers at time of foot contact of leading leg) (Fig. 2A). This difference was normalized by the step lengths sum to account for step length differences across subjects. A step symmetry value of 0 would indicate that step lengths are equal, a positive value would indicate that the leg on the fast belt is taking longer steps, and a negative value would indicate that the leg on the slow belt is taking longer steps.
The temporal parameter was the phase shift between the two legs. We computed the cross-correlation between limb angle trajectories during one full step cycle for each leg (Fig. 2B). Limb angle was defined as the angle between the vertical and the vector from hip to ankle on the $x, y$ plane (Fig. $2 B$ ). Phase shift was the lag or lead time leading to maximum correlation between limb angle trajectories (Fig. $2 B$, black and gray lines). A phase shift value of 0.5 would indicate that legs are moving in anti-phase (Fig. $2 \mathrm{~B}$, plot in top left corner of panel). To correct for subjects' biases we subtracted the phase shift during the baseline period from all other periods. Consequently, a value of 0 indicates that legs are moving in anti-phase, positive phase shifts indicate that the fast leg is phase advanced relative to the slow leg, and negative phase shifts indicate that the fast leg is lagging the slow leg.

We quantified the magnitude of adaptation on the treadmill ( $\left.\mathrm{TM}_{\text {learning }}\right)$, its transfer to overground walking $\left(\mathrm{OG}_{\text {transfer }}\right)$, and subsequent washout of the adaptation when returning to the treadmill $\left(\mathrm{TM}_{\text {washout }}\right)$ in the following manner. $\mathrm{TM}_{\text {learning }}$ was defined as the size of the aftereffects during catch trial, corrected for any baseline biases (Eq. 1). OG $\mathrm{G}_{\text {transfer }}$ and $\mathrm{TM}_{\text {washout }}$ were similarly corrected for baseline and then expressed as a percentage of $\mathrm{TM}_{\text {learning }}$ (Eqs. 2 and 3):

$$
\begin{gathered}
\mathrm{TM}_{\text {learning }}=\mathrm{TM}_{\text {catch }}-\mathrm{TM}_{\text {baseline }} \\
\mathrm{OG}_{\text {transfer }}=\frac{\mathrm{OG}_{\mathrm{after}}-\mathrm{OG}_{\text {baseline }}}{\mathrm{TM}_{\text {learning }}} * 100 . \\
\mathrm{TM}_{\text {washout }}=\left(1-\frac{\mathrm{TM}_{\mathrm{after}}-\mathrm{TM}_{\text {baseline }}}{\mathrm{TM}_{\text {learning }}}\right) * 100 .
\end{gathered}
$$

$\mathrm{OG}_{\text {baseline }}$ and $\mathrm{TM}_{\text {baseline }}$ are the mean of all strides in the overground and treadmill baseline periods, respectively. $\mathrm{TM}_{\text {catch }}$ is the mean aftereffect of the first 3 strides during the catch trial period. OG $\mathrm{after}_{\text {and }} \mathrm{TM}_{\mathrm{after}}$ are the mean aftereffect of the first 3 strides during postadaptation periods when subjects walked off and on the treadmill, respectively.

We quantified the variability of stepping across conditions by measuring stepping cadence, which is defined as the inverse of the timing between heel strikes of the two legs. Stepping cadence was used, rather than symmetry measures, because we wanted to characterize the variability in the movement of each leg. Also, stepping cadence was used, rather than other leg-specific parameters such as step length, because it is a measure less sensitive to subject's ability to adapt (Reisman et al., 2005). Thus its variance represents better the variability in subjects' behavior during adaptation rather than differences in the extent of adaptation across subjects. The overall variance in each subject's motor behavior was calculated using the stepping cadence during baseline and adaptation. We subtracted the means of the left and right leg distributions to center them around zero and calculated an overall variance of the merged distributions.

\section{Statistical analysis}

One-way ANOVA was used to compare learning, transfer, and washout across experimental groups; post hoc analyses were performed using the Fisher's LSD significant different test. We also used multiple-regression to determine how variability in stepping and sensory condition during adaptation or during testing affected learning $\left(\mathrm{TM}_{\text {learning }}\right)$, transfer $\left(\mathrm{OG}_{\text {transfer }}\right)$, and washout $\left(\mathrm{TM}_{\text {washout }}\right)$. The predicted learning, transfer, and washout variables were obtained as the linear combination of variability of stepping $\left(\sigma^{2}\right)$, sensory condition during adaptation $\left(V_{\text {training }}\right)$, and the sensory condition during testing $\left(V_{\text {testing }}\right)$. The categorical regressors $V_{\text {training }}$ and $V_{\text {testing }}$ were set to 1 when subjects were trained or tested without vision and 0 when they were trained or tested with vision. Note that $V_{\text {testing }}$ for predicted learning $\left(\widehat{\mathrm{TM}}_{\text {learning }}\right)$ were determined by the sensory condition during catch whereas $V_{\text {testing }}$ for predicted transfer $\left(\widehat{O G}_{\text {transfer }}\right)$ and washout ( $\widehat{\mathrm{TM}}_{\text {washout }}$ ) were determined by the sensory condition during overground walking. Stated formally:

$$
\widehat{\mathrm{TM}}_{\text {learning }}=b_{0}+b_{1} \sigma 2+b_{2} V_{\text {training }}+b_{3} V_{\text {testing, }}
$$


A Step symmetry (spatial)

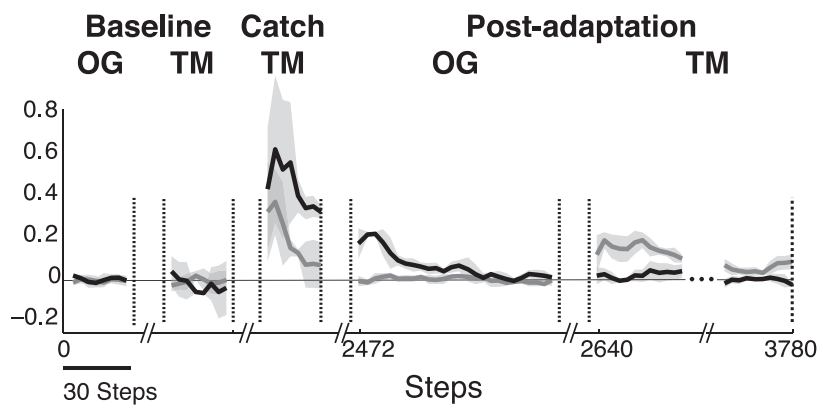

B Phase shift symmetry (temporal)

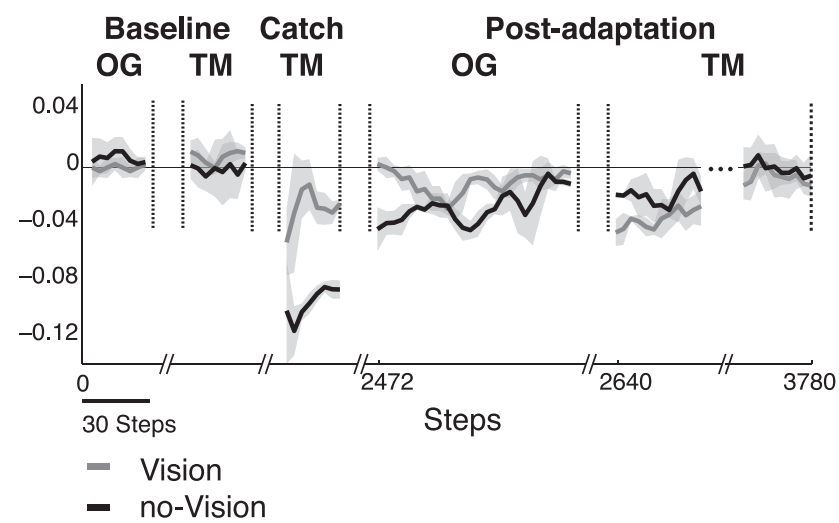

Figure 3. Symmetry in spatial and temporal gait features of sample subjects of the vision and no-vision group when walking on the treadmill (i.e., TM) and over ground (i.e., OG) during pre- and postadaptation. $\boldsymbol{A}$, Spatial symmetry (i.e., symmetry in step lengths of the two legs) when walking on the treadmill and over ground during baseline, catch, and postadaptation periods. Behavior of two sample subjects is shown: one walking with vision (gray trace) and one walking without vision (black trace). Lines represent the running average using a 3 step window \pm SD (shaded area). No differences in spatial symmetry were observed preadaptation when subjects walked with and without vision on the treadmill or over ground. However, more learning was observed in subject trained without vision than in subject trained with vision, as indicated by the differences in aftereffects during the catch trial on the treadmill. Similarly, more transfer of aftereffects to overground walking is observed in subject walking without vision than in subject walking with vision, as indicated by the larger aftereffects during postadaptation OG. Also, more washout of aftereffects is observed in subject walking without vision than in subject walking with vision, as indicated by the differences in remaining aftereffects when subjects returned to the treadmill. $\boldsymbol{B}$, Temporal symmetry (i.e., symmetry in heel strike timing of one leg relative to the other) when walking on the treadmill and over ground during baseline, catch, and postadaptation periods. Vision had similar effects on temporal symmetry to those shown for spatial symmetry. Subject that walked without vision had larger temporal aftereffects, more transfer, and more washout than subject that walked with vision.

where

$$
V_{\text {testing/training }}=\left\{\begin{array}{l}
0 \text { if tested/trained with vision } \\
1 \text { if tested/trained without vision. }
\end{array}\right\}
$$

Regression equations of the same form were used to determine $\widehat{\mathrm{OG}}_{\text {transfer }}$ and $\widehat{\mathrm{TM}}_{\text {washout }}$.

We used $p<0.05$ as a measure of significance for all statistical analyses, which were completed using Statistica (StatSoft) software.

\section{Results}

Removing context-specific visual cues increases learning, transfer, and washout of split-belt adaptation

We found that context-specific visual cues were a strong modulator of split-belt walking adaptation and its transfer to overground walking. Figure 3 shows single subject examples of

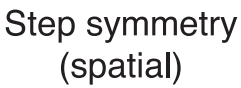

\section{Phase shift (temporal)}

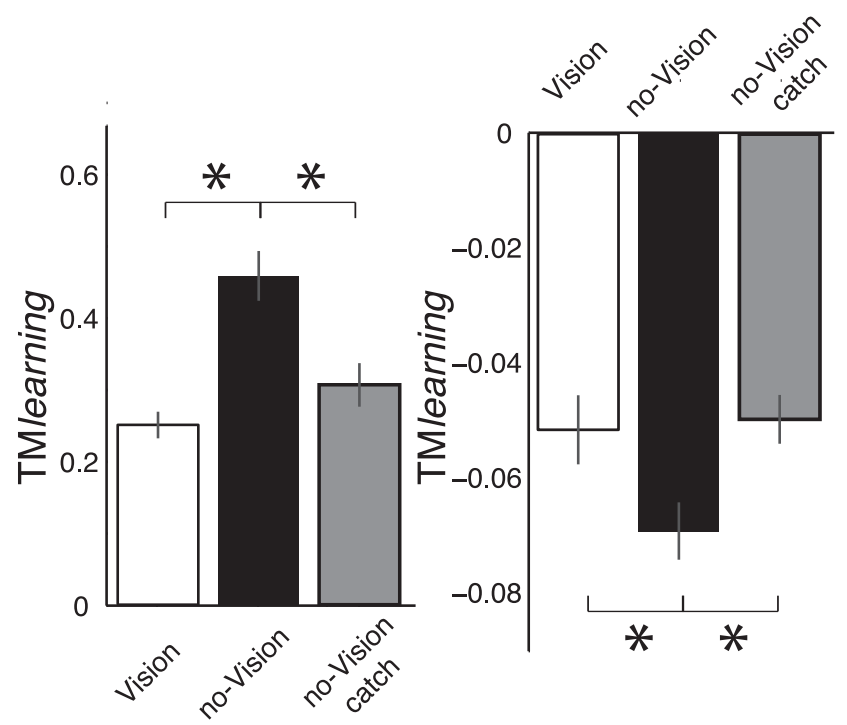

Figure 4. Aftereffects on treadmill during catch trial for all groups. Subjects that trained without vision had significantly larger spatial and temporal aftereffects than subjects that trained with vision. Moreover, these differences were not due to the lack of vision during the catch trial. Since a group of subjects that was trained with vision but close their eyes during the catch (gray bar; no-vision catch group) had similar aftereffects to those subjects trained with vision (white bar) and significantly smaller aftereffects that those subjects trained without vision (black bar). Bars' height indicates the averaged $\mathrm{TM}_{\text {learning }}$ across subjects $\pm \mathrm{SE}$. ${ }^{*} p<$ 0.05 (significant difference).

averaged step-by-step data across 3 steps from the vision and no-vision groups for step symmetry and phase shift. Select periods of the experiment are shown to emphasize the differences in aftereffects on and off of the treadmill. When vision was removed, we observed larger aftereffects during catch trials on the treadmill for both step symmetry and phase shift. Overground transfer of the aftereffect and washout of aftereffects when returning to the treadmill was also larger in the no-vision group. Since we observed differences in learning across subjects, the extent of transfer and washout were normalized with respect to each subject's learning $\left(\mathrm{TM}_{\text {learning }}\right)$ before comparing them across subjects (see Materials and Methods).

The larger aftereffects when subjects are trained without vision are reflected in the group data. Figure 4 shows data for TMlearning for the vision, no-vision, and no-vision catch groups during the catch trial. Recall that the no-vision catch group was added so that we could determine whether the increased aftereffect for treadmill learning in the no-vision condition was due to performing the catch trial without vision or adapting without vision. We found a significant effect of condition on treadmill learning for step symmetry $\left(F_{(2,36)}=15.74, p<0.001\right)$ and phase shift $\left(F_{(2,36)}=3.81, p=0.03\right)$. Subjects in the no-vision group showed greater treadmill learning (i.e., $\mathrm{TM}_{\text {learning }}$ ) than the vision group and no-vision catch for step symmetry $(p<0.003)$ and phase shift $(p<0.042)$. Note, the no-vision catch group was not statistically different from the vision group ( $p=0.25$ for step symmetry and $p=0.85$ for phase shift). This strongly suggests that the improvement in the no-vision group is due to adapting without vision rather than removing vision during the catch trial.

Removing context-specific visual cues increased transfer of learning to overground walking $\left(F_{(3,35)}=4.19, p=0.01\right.$ for step symmetry and $F_{(3,35)}=8.87, p<0.001$ for phase shift) and sub- 


\section{Transfer}
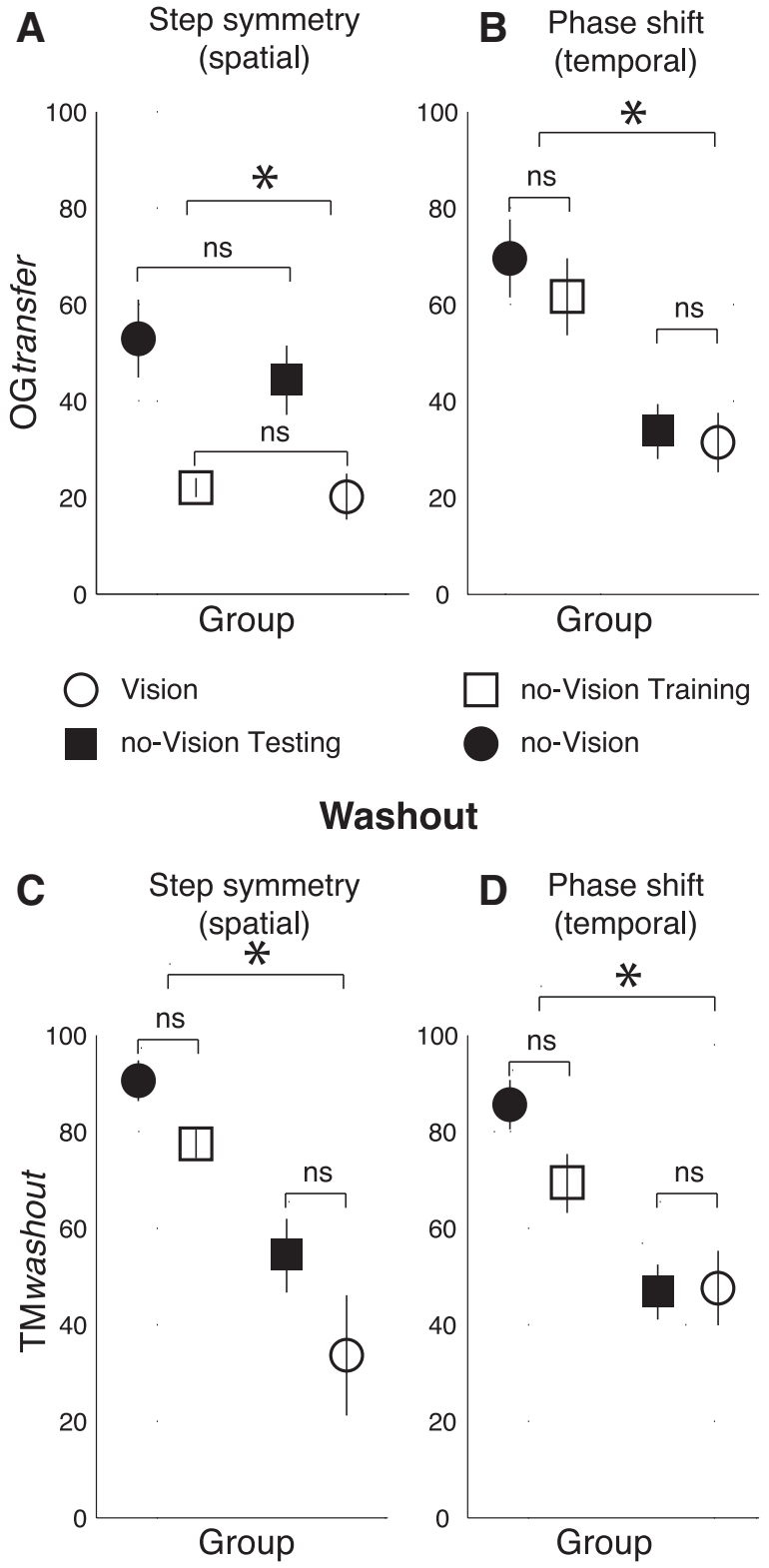

Focusing on experiment 1 , it is clear that transfer was better for the no-vision (black solid) versus vision (open), groups for step symmetry (Fig. $5 A, p=0.01$ ) and phase shift (Fig. $5 B, p<0.001$ ). Note also that the overall transfer is smaller for step symmetry than for phase shift, suggesting that temporal learning is more general. Figure $5 C$ shows that there was near complete washout of treadmill aftereffects following overground walking in the novision versus vision groups for step symmetry $(p<0.001)$ and substantial washout for phase shift $(p<0.001)$ (Fig. 5D). This further supports that subjects were relying on the same neural circuits for treadmill and overground walking in the no-vision condition.

\section{Removing context-specific visual cues in the training versus the testing context}

The strong effects of vision on transfer could be due to the removal of vision during adaptation (i.e., training) and/or the removal of vision during overground walking (i.e., overground testing). To address this question we performed experiment 2 where one group was trained without vision and then tested over ground with vision, and vice versa for the other group. Figure $5 \mathrm{~A}$ shows that removing vision during training did not improve transfer of step symmetry, whereas removing vision during overground testing did. In fact, transfer of step symmetry was just as strong in the no-vision testing group compared with the novision group in experiment $1(p=0.70)$. In contrast, transfer of temporal aftereffects was improved when we removed visual cues during training but not overground testing. Figure $5 B$ shows that $O G_{\text {transfer }}$ of phase shift in the no-vision training group was just as strong as in the no-vision group ( $p=0.28)$, and significantly larger than that of the groups trained with vision $(p<0.001)$.

Figure 5, $C$ and $D$, shows how much of the treadmill aftereffect was washed out following overground walking. The amount of washout is another assessment of the amount of transfer (i.e., the more transfer, the more washout). Figure $5 D$ shows that this was true for the washout of temporal aftereffects: $\mathrm{TM}_{\text {washout }}$ of phase was largest in groups with largest $O_{\text {transfer }}$ shown in Figure $5 B$. Specifically, the no-vision training group showed large amounts of washout similar to the no-vision group (for step symmetry $p=$ 0.31 and for phase shift $p=0.28$ ) but was different from that of the groups trained with vision (no-vision testing and vision groups; $p<0.046$ for step symmetry and $p<0.01$ for phase shift).

On the other hand, Figure $5 C$ shows that washout for step symmetry did not follow the expected pattern: $\mathrm{TM}_{\text {washout }}$ of step symmetry was not largest in groups with the largest $\mathrm{OG}_{\text {transfer }}$ shown in Figure 5A. Specifically, the no-vision testing showed small amounts of washout similar to the vision group (for step symmetry $p=0.38$ and for phase shift $p=0.65$ ), but different from the groups trained without vision (no-vision training and no-vision groups; $p<0.046$ for step symmetry and $p<0.01$ for phase shift). This suggests that the absence of visual context cues during training on the treadmill is what allows treadmill aftereffects to washout. If context cues are present during training, it is difficult to wash out the treadmill aftereffects.

\section{Variable behavior during adaptation increases learning}

Variability in the behavior had an effect on the magnitude of adaptation but not on the transfer or washout of the adaptation. Figure 6 shows the observed $\mathrm{TM}_{\text {learning }}$ values as a function of variance in stepping cadence for each subject. Regardless of the testing or training sensory conditions, subjects who were more variable had larger aftereffects during the catch trial in spatial and temporal parameters sequent washout of aftereffects when returning to the treadmill $\left(F_{(3,35)}=5.94, p=0.002\right.$ for step symmetry and $F_{(3,35)}=7.47$, $p<0.001$ for phase shift). Figure 5 shows overground transfer, $\mathrm{OG}_{\text {transfer }}$, for the no-vision and vision groups in experiment 1 (and also for conditions from experiment 2 described below). 


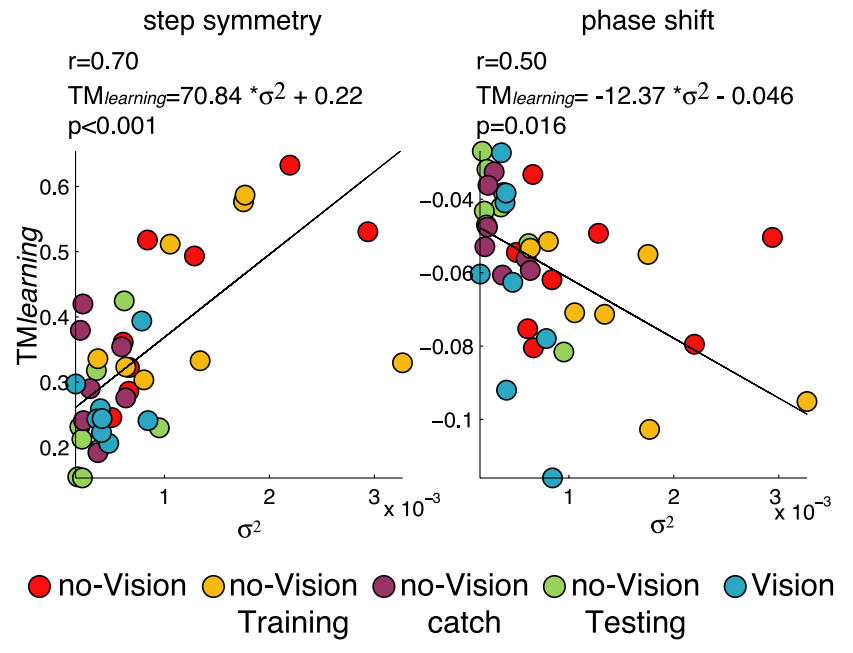

Figure 6. Scatter plots showing the relationship between variability in stepping and $\mathrm{TM}_{\text {learning. }}$ Colors indicate the different sensory conditions. Multiple regression was used to explore the effects of variability, training, and testing sensory conditions (i.e., 3 regressors) on adaptation magnitude ( $\left.\mathrm{TM}_{\text {learning }}\right)$. Regression equations with significant factors are included in both panels. Variability in behavior was the only significant factor that predicted the magnitude of spatial and temporal adaptation effects. The magnitude of adaptation was positively related to the subjects' behavior variability: the more variable were subjects during adaptation, the more learning (i.e., bigger aftereffects during catch trial).

$\left(\widehat{\mathrm{TM}}_{\text {learning }}=70.84 * \sigma^{2}-0.22, F_{(3,35)}=11.28, p<0.001\right.$ for step symmetry and $\widehat{\mathrm{TM}}_{\text {learning }}=-12.37 * \sigma^{2}-0.046, F_{(3,35)}=3.93, p=$ 0.016 for phase shift) (Fig. 6). On the other hand, sensory condition was the only significant regressor of $\mathrm{OG}_{\text {transfer. }}$. Consistent with data shown in Figure 5, the testing sensory condition was related to $\mathrm{OG}_{\text {transfer }}$ for step symmetry $\left(\widehat{\mathrm{OG}}_{\text {transfer }}=25.83 * V_{\text {testing }}+18.26, F_{(3,35)}=4.02, p=\right.$ $0.015)$ and the training sensory condition was related to $\mathrm{OG}_{\text {transfer }}$ for phase shift $\left(\widehat{O G}_{\text {transfer }}=34.49 * V_{\text {training }}+28.95, F_{(3,35)}=8.06, p<\right.$ $0.001)$. Finally, the training sensory condition was the only significant regressor of $\mathrm{TM}_{\text {washout }}\left(\widehat{\mathrm{TM}}_{\text {washout }}=41.12 * V_{\text {training }}+43.29\right.$, $F_{(3,35)}=7.27, p<0.001$ for step symmetry and $\widehat{\mathrm{TM}}_{\text {washout }}=28.98 *$ $V_{\text {training }}+35.94, F_{(3,35)}=7.35, p<0.001$ for phase shift). These results indicate that the main effect for transfer and washout of treadmill aftereffects has to do with sensory context.

\section{Discussion}

We found that removing visual contextual cues increased transfer of treadmill adaptation to natural walking and degraded the ability to generate a device-specific internal model for the treadmill. Further, the transfer of temporal and spatial aftereffects was dissociated when eliminating vision at different times within the experiment. Overall, these results demonstrate that vision is important for the formation of context-specific internal models for walking. We think that this is because visual cues allow us to attach newly learned walking patterns to specific environments or walking conditions.

\section{Adaptation is strengthened when vision is removed}

Why did removing vision during treadmill training strengthen adaptation-i.e., lead to a larger catch trial aftereffect? We ruled out the possibility that this was due to a balance perturbation effect from having eyes closed during the catch trial (Fig. 4). Instead, we suggest that removing vision (1) led to more variable behavior, which may in turn increase sensitivity to the error driv- ing adaptation and also (2) altered sensory weightings in a manner that benefits adaptation.

The first interpretation is supported by the theory that the nervous system acts as a Bayesian estimator, which learns more from error when it is certain of the sensory information encoding the error and uncertain about its internal predictions of movement parameters to accomplish the goal (Korenberg and Ghahramani, 2002; Burge et al., 2008; Wei and Körding, 2009, 2010). We interpret the observed increase in variability as being related to greater uncertainty in internal movement predictions, such that subjects would consequently learn more from their errors. This explanation is supported by our regression analysis showing that the amount of adaptation is best explained by the variability of stepping frequency - the more variable their behavior, the more subjects learn. Similar effects have been observed in forms of motor adaptation involving arm control (Burge et al., 2008; Wei and Körding, 2009, 2010). Our data may suggest that this is a general principle that applies to control of very different movement types (i.e., walking versus reaching).

Moreover, removing vision might have strengthened adaptation because the gain of the sensory information driving the adaptation increased due to sensory reweighting. It has been demonstrated that proprioceptive gain increases when vision is occluded in standing balance (Kiemel et al., 2002; Peterka and Loughlin, 2004), and we suggest that the same happened for subjects that walked without vision. Proprioception is an important sensory modality encoding errors in motor adaptation to sustained dynamic perturbations like the one presented here (Krakauer et al., 1999) and it is necessary for other forms of locomotor adaptation (Bunday and Bronstein, 2009). Therefore, increasing the gain of proprioception when removing vision could contribute to more robust motor learning.

\section{Removing vision affects transfer of learning}

We found that visual cues are normally important for generating separate internal models for walking in different environments. When subjects had full vision throughout the experiment, we saw limited transfer of temporal and spatial aftereffects to overground walking and strong aftereffects when subjects returned to the treadmill. Therefore, vision provided some contextual information leading to the formation of a device-specific internal model. This was true despite the generality of the neural circuitry encoding different forms of locomotion (Shik et al., 1969; Stein et al., 1998; Dietz, 2003; Kiehn, 2006; McCrea and Rybak, 2008). Thus, while locomotor patterns may use shared circuitry at the spinal level, multiple internal models for walking on different contexts can easily be developed within higher centers $(\mathrm{McVea}$ and Pearson, 2007), such as the cerebellum (Morton and Bastian, 2006). This is also consistent with studies showing separate internal model formation in eye and upper body movements (Gandolfo et al., 1996; Osu et al., 2004; Cothros et al., 2009; Herman et al., 2009; Howard et al., 2010). Together, these findings represent evidence of the computational similarities between different types of movements regarding contextual learning.

In contrast, removing vision during all periods of the experiment doubled the transfer of temporal and spatial aftereffects. It had a similar effect on the amount of washout on the treadmill$70-80 \%$ of the aftereffect was gone. These results indicate that closing the eyes led to an adaptation not tied to the training device. One possible explanation for this is that removing vision during training increased the learning encoded in intrinsic coordinates (i.e., attached to body), which is more likely to be performed with the body across different environments than 
learning encoded in extrinsic coordinates (i.e., attached to the environment). Proprioception is the primary sensory modality encoding learning in intrinsic coordinates rather than in extrinsic coordinates (Shadmehr and Mussa-Ivaldi, 1994). Moreover, the gain of proprioception increases when subjects walked without vision due to sensory reweighting (Kiemel et al., 2002; Peterka and Loughlin, 2004). Therefore, in addition to increasing learning, the up-weighted proprioceptive gain when trained without vision could also lead to increases in the adaptation in intrinsic versus extrinsic coordinates. As a consequence, learning effects should more easily transfer with the body across different environments. This is consistent with a recent reaching study showing that adaptation to gradual perturbations encoded in intrinsic coordinates (Malfait and Ostry, 2004) increases transfer of reaching adaptation to movements without the device (Kluzik et al., 2008).

Another possibility is that removing vision during training changed the subjects' perception of the source of error during adaptation (i.e., credit assignment) in a manner that benefits generalization. It has been proposed that credit assignment, or the ability to assign errors to the environment or the body, underlies the generalization of learning (Berniker and Kodning, 2008). If the source of an error were estimated to be the environment, one would adapt and apply the learning only to that particular situation. Conversely if the source of error were estimated to be self-induced, one would adapt and apply the learning to movements in any other environments. Interpreted in this way, our results suggest that when removing the visual-proprioceptive mismatch specific to treadmills, the subjects' nervous system became less "aware" that they were walking on a treadmill. Consequently, the errors that they experienced were more easily attributed to their own movements and less to that particular walking environment; leading to more transfer of the acquired learning to overground walking.

\section{Removing vision affects the relative contribution of internal model to foot placement control}

Since vision had an effect on transfer, we tested the relative importance of removing vision during split-belt training versus overground testing. We expected that transfer would only improve when we removed vision during training, since this would change how the learned pattern was encoded. This was only true, however, for the temporal aftereffect-it transferred more when vision was removed only in training, and did not transfer more when vision was removed only in testing. In contrast, the transfer of spatial aftereffects showed the opposite pattern. Spatial transfer was small when trained without vision, and large when tested without vision.

A possible explanation for these results is that manipulating vision during testing allows people to use online visual feedback to override internal model predictions controlling step location (spatial aftereffects) but not step timing (temporal aftereffects). It is known that movement control is achieved through the contribution of online feedback and feedforward predictions based on internal models (Wolpert et al., 1995). Although split-belt walking changes internal model predictions of both spatial and temporal features of gait (Reisman et al., 2005), online visual feedback may specifically contribute to the control of spatial aspects of gait (Marigold and Patla, 2008). Therefore, testing with vision would increase the reliance on online feedback control for foot placement.

In contrast, testing without vision would increase reliance on the adapted internal model, leading to larger transfer of spatial aftereffects. This is in line with other studies in which feedforward control was modified but changes in the behavior could only be observed when the contribution of online feedback was diminished by removing vision (Gordon et al., 1995).

In addition, our recent work also suggests that spatial and temporal adaptation may be controlled by different processes. We found that people can alter the time course of one without changing the other-e.g., spatial and temporal aspects of walking can adapt at different rates during split-belt training (Malone and Bastian, 2010). Thus, it may be that different neural structures are involved in adapting spatial and temporal control, and therefore they may also show different sensitivities to visual input affecting transfer.

\section{Clinical implications}

Promising studies have suggested that split-belt adaptation could help rehabilitate subjects with asymmetric gait (Reisman et al., 2007; Choi et al., 2009). It is therefore critical to understand what factors can increase the transfer of movements learned in the device to natural situations. Here we showed that device-specific learning can become more general if salient cues about the training environment are diminished or manipulated to match the "real world". Therefore, we are exploring alternative methods to manipulate visual context (e.g., optic flow matching natural walking) for the purpose of improving walking adaptation and transfer in patients with gait asymmetry.

\section{References}

Berniker M, Kording K (2008) Estimating the sources of motor errors for adaptation and generalization. Nat Neurosci 11:1454-1461.

Bunday KL, Bronstein AM (2009) Locomotor adaptation and aftereffects in patients with reduced somatosensory input due to peripheral neuropathy. J Neurophysiol 102:3119-3128.

Burge J, Ernst MO, Banks MS (2008) The statistical determinants of adaptation rate in human reaching. J Vis 8:20.1-19.

Choi JT, Bastian AJ (2007) Adaptation reveals independent control networks for human walking. Nat Neurosci 10:1055-1062.

Choi JT, Vining EP, Reisman DS, Bastian AJ (2009) Walking flexibility after hemispherectomy: split-belt treadmill adaptation and feedback control. Brain 132:722-733.

Cothros N, Wong JD, Gribble PL (2006) Are there distinct neural representations of object and limb dynamics? Exp Brain Res 173:689-697.

Cothros N, Wong J, Gribble PL (2009) Visual cues signaling object grasp reduce interference in motor learning. J Neurophysiol 102:2112-2120.

Dietz V (2003) Spinal cord pattern generators for locomotion. Clin Neurophysiol 114:1379-1389.

Gandolfo F, Mussa-Ivaldi FA, Bizzi E (1996) Motor learning by field approximation. Proc Natl Acad Sci U S A 93:3843-3846.

Gordon CR, Fletcher WA, Melvill Jones G, Block EW (1995) Adaptive plasticity in the control of locomotor trajectory. Exp Brain Res 102:540-545.

Haruno M, Wolpert DM, Kawato M (2001) Mosaic model for sensorimotor learning and control. Neural Comput 13:2201-2220.

Herman JP, Harwood MR, Wallman J (2009) Saccade adaptation specific to visual context. J Neurophysiol 101:1713-1721.

Howard IS, Ingram JN, Wolpert DM (2010) Context-dependent partitioning of motor learning in bimanual movements. J Neurophysiol 104:2082-2091.

Imamizu H, Kuroda T, Miyauchi S, Yoshioka T, Kawato M (2003) Modular organization of internal models of tools in the human cerebellum. Proc Natl Acad Sci U S A 100:5461-5466.

Kiehn O (2006) Locomotor circuits in the mammalian spinal cord. Annu Rev Neurosci 29:279-306.

Kiemel T, Oie KS, Jeka JJ (2002) Multisensory fusion and the stochastic structure of postural sway. Biol Cybern 87:262-277.

Kluzik J, Diedrichsen J, Shadmehr R, Bastian AJ (2008) Reach adaptation: what determines whether we learn an internal model of the tool or adapt the model of our arm? J Neurophysiol 100:1455-1464.

Korenberg A, Ghahramani Z (2002) A Bayesian view of motor adaptation. Curr Psychol Cogn 21:537-564.

Krakauer JW, Ghilardi MF, Ghez C (1999) Independent learning of internal 
models for kinematic and dynamic control of reaching. Nat Neurosci 2:1026-1031.

Lee JY, Schweighofer N (2009) Dual adaptation supports a parallel architecture of motor memory. J Neurosci 29:10396-10404.

Malfait N, Ostry DJ (2004) Is interlimb transfer of force-field adaptation a cognitive response to the sudden introduction of load? J Neurosci 24:8084-8089.

Malone LA, Bastian AJ (2010) Thinking about walking: effects of conscious correction versus distraction on locomotor adaptation. J Neurophysiol 103:1954-1962.

Marigold DS, Patla AE (2008) Visual information from the lower visual field is important for walking across multi-surface terrain. Exp Brain Res 188:23-31.

McCrea DA, Rybak IA (2008) Organization of mammalian locomotor rhythm and pattern generation. Brain Res Rev 57:134-146.

McVea D, Pearson K (2007) Contextual learning and obstacle memory in the walking cat. Integr Comp Biol 47:457-464.

Morton SM, Bastian AJ (2006) Cerebellar contributions to locomotor adaptations during splitbelt treadmill walking. J Neurosci 26:9107-9116.

Osu R, Hirai S, Yoshioka T, Kawato M (2004) Random presentation enables subjects to adapt to two opposing forces on the hand. Nat Neurosci 7:111-112.

Peterka RJ, Loughlin PJ (2004) Dynamic regulation of sensorimotor integration in human postural control. J Neurophysiol 91:410-423.

Reisman DS, Block HJ, Bastian AJ (2005) Interlimb coordination during locomotion: what can be adapted and stored? J Neurophysiol 94:24032415 .
Reisman DS, Wityk R, Silver K, Bastian AJ (2007) Locomotor adaptation on a split-belt treadmill can improve walking symmetry post-stroke. Brain 130:1861-1872.

Reisman DS, Wityk R, Silver K, Bastian AJ (2009) Split-belt treadmill adaptation transfers to overground walking in persons poststroke. Neurorehabil Neural Repair 23:735-744.

Shadmehr R, Mussa-Ivaldi FA (1994) Adaptive representation of dynamics during learning of a motor task. J Neurosci 14:3208-3224.

Shelhamer M, Aboukhalil A, Clendaniel R (2005) Context-specific adaptation of saccade gain is enhanced with rest intervals between changes in context state. Ann N Y Acad Sci 1039:166-175.

Shik ML, Severin FV, Orlovsky GN (1969) Control of walking and running by means of electrical stimulation of the mesencephalon. Electroencephalogr Clin Neurophysiol 26:549.

Stein PS, McCullough ML, Currie SN (1998) Spinal motor patterns in the turtle. Ann N Y Acad Sci 860:142-154.

Wei K, Körding K (2009) Relevance of error: what drives motor adaptation? J Neurophysiol 101:655-664.

Wei K, Körding K (2010) Uncertainty of feedback and state estimation determines the speed of motor adaptation. Front Comput Neurosci 4:11.

Wolpert D, Miall R, Kawato M (1998) Internal models in the cerebellum. Trends Cogn Sci 2:338-347.

Wolpert DM, Kawato M (1998) Multiple paired forward and inverse models for motor control. Neural Netw 11:1317-1329.

Wolpert DM, Ghahramani Z, Jordan MI (1995) An internal model for sensorimotor integration. Science 269:1880-1882. 